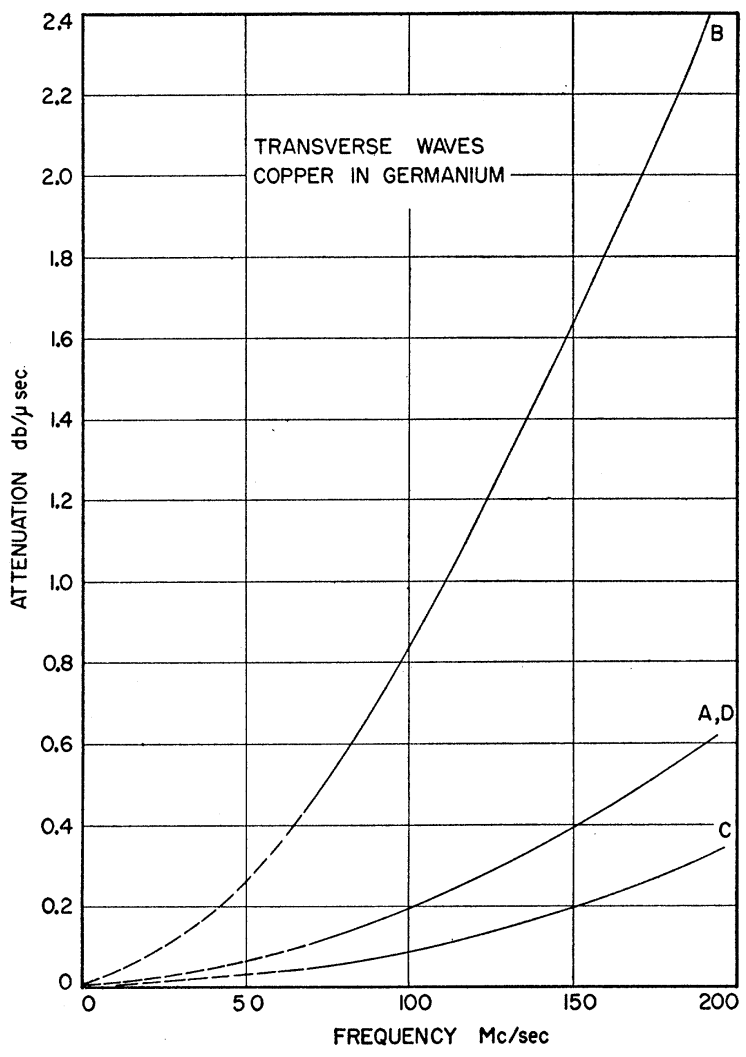

Fig. 2. Attenuation as a function of copper present. Transverse wave attenuation. (A) crystal as originally measured; (B) after heating at $800^{\circ} \mathrm{C}$ in vacuum; (C) after diffusion of copper into specimen; (D) after reheating in vacuum.

be shown, however, that because the solubility of copper in germanium is quite low (maximum solubility ${ }^{5}$ is about $4 \times 10^{16}$ atoms/cc), there is, excluding the possibility of precipitation effects, not sufficient copper on the dislocations to account for the magnitude of the attenuation change observed. It seems that the precipitation of copper on dislocations is responsible for the large observed changes; additional evidence for this can be found in resistivity measurements. ${ }^{6}$

Experiments are under way to determine quantitatively the effect of precipitated copper in connection with the observed effects. Attenuation changes have already been shown to be a sensitive method of indicating precipitation effects in internal oxidation in dilute copper alloys. It is expected that in this case with copper in germanium the attenuation changes will also prove to be a sensitive method of studying precipitation effects.

* This work was supported in part by the Solid State Devices Section of the U. S. Army Signal Corps.

1 A. Granato and R. Truell, J. Appl. Phys. (to be published).

2 A. Granato and K. Lücke, J. Appl. Phys. (to be published).

3 G. Finn, Phys. Rev. 91, 754 (1953).

${ }^{4} \mathrm{~K}$. Blodgett, J. Appl. Phys. 26, 1520 (1954).

5 Fuller, Struthers, Ditzenberger, and Wolfstirn, Phys. Rev. 93, 1182 (1954).

${ }^{6}$ R, A. Logan, Phys. Rev, 100, 615 (1955).

\section{Empirical Determination of Nuclear Moments of Inertia and Intrinsic Quadrupole Moments}

\author{
E. D. Klema and R. K. Osborn
}

Oak Ridge National Laboratory, Oak Ridge, Tennessee

(Received May 28, 1956)

$\mathrm{A}^{\mathrm{N}}$ $\mathrm{N}$ empirical parameter, $k$, has been found which enables one to calculate the fractional nuclear mass or charge which takes part in collective phenomena for a given even-even nucleus. This parameter is defined as the ratio of the number of particles outside of filled major shells (both protons and neutrons) or the number of holes, whichever is smaller, to the total number of particles in the nucleus.

If one assumes that the fractional nuclear mass, $k M$, where $M$ is the mass of the nucleus, rotates about an axis at right angles to the axis of symmetry, as an axially-symmetric rigid rotator with uniform mass distribution, the moment of inertia is given by $\Lambda_{x}$ $=\frac{1}{5} k M R_{0}{ }^{2}\left(\gamma^{2}+\alpha^{2}\right)$, where $\gamma$ is the distance along the axis of symmetry in units of $R_{0}, \alpha$ is the distance along the axis at right angles to the axis of symmetry, and $R_{0}$ is the undeformed nuclear radius, here taken to be $1.44 A^{\frac{1}{3}} \times 10^{-13} \mathrm{~cm}$. The energy of such a rigid rotator with angular momentum $\lambda$ is given by $E_{\lambda}=\left(\hbar^{2} / 2 \Lambda_{x}\right)$ $\times \lambda(\lambda+1)$. The intrinsic quadrupole moment, $Q_{0}$, is defined by $\int \rho r^{2}\left(3 \cos ^{2} \theta-1\right) d \tau$. For the case of an axially-symmetric rigid rotator with uniform charge distribution, $Q_{0}=\frac{2}{5} Z R_{0}^{2}\left(\gamma^{2}-\alpha^{2}\right)$. Under the assumption that the deformation is volume-preserving, $\alpha^{2} \gamma=1$.

Thus, using the above relationships together with the known number of nucleons in a given nucleus and the measured energy of its first-excited $2^{+}$state (when it is known to be a member of a pure rotational sequence), one can obtain the intrinsic quadrupole

TABLE I. Intrinsic quadrupole moments, $Q_{0}\left(E_{2^{+}}\right)$, and shape factors, $\gamma$, obtained from the energies of first-excited states; and intrinsic quadrupole moments, $Q_{0}(\mathrm{~B}-\mathrm{M}) / k$, obtained from transition data. The moments are given in units of $10^{-24} \mathrm{~cm}^{2}$.

\begin{tabular}{|c|c|c|c|c|c|c|c|}
\hline Nucleus & $k$ & $\begin{array}{l}E_{2^{+}} \\
(\mathrm{kev})\end{array}$ & $\gamma$ & $Q_{0}\left(E_{2}+\right)$ & $Q_{0}(B-M)$ & $\frac{Q_{0}(\mathrm{~B}-\mathrm{M})}{k}$ & $\frac{Q_{0}(\mathrm{~B}-\mathrm{M}) / k}{Q_{0}\left(E_{2^{2}}\right)}$ \\
\hline${ }_{64} \mathrm{Gd}^{154}$ & 0.1428 & 123 & 1.83 & 42.7 & 6.6 & 46 & 1.08 \\
\hline $\mathrm{Gd}^{156}$ & 0.1538 & 89 & 2.10 & 60.5 & 8.4 & 55 & 0.91 \\
\hline $\mathrm{Gd}^{158}$ & 0.1646 & 79 & 2.14 & 63.8 & 9.3 & 56 & 0.88 \\
\hline $\mathrm{Gd}^{160}$ & 0.1750 & 76 & 2.08 & 60.2 & 9.8 & 56 & 0.93 \\
\hline${ }_{66} \mathrm{Dy}^{160}$ & 0.1750 & 86.2 & 1.93 & 51.7 & 7.1 & 41 & 0.79 \\
\hline Dy $^{162}$ & 0.1852 & 82 & 1.90 & 50.2 & 7.9 & 43 & 0.86 \\
\hline $\mathrm{Dy}^{164}$ & 0.1951 & 73 & 1.95 & 54.0 & 9.2 & 47 & 0.87 \\
\hline${ }_{68} \mathrm{Er}^{164}$ & 0.1707 & 91 & 1.85 & 48.7 & 7.3 & 43 & 0.88 \\
\hline $\operatorname{Err}^{166}$ & 0.1807 & 80 & 1.91 & 53.2 & & & \\
\hline${ }_{70} \mathrm{Yb}^{170}$ & 0.1765 & 84.1 & 1.83 & 49.9 & 7.1 & 40 & 0.80 \\
\hline${ }_{72} \mathrm{Hf}{ }^{176}$ & 0.1818 & 87 & 1.69 & 42.5 & 7.1 & 39 & 0.92 \\
\hline $\mathrm{Hf}^{178}$ & 0.1685 & 90 & 1.72 & 44.9 & 7.7 & 46 & 1.02 \\
\hline $\mathrm{Hf}^{180}$ & 0.1556 & 93 & 1.75 & 47.4 & 7.2 & 46 & 0.97 \\
\hline${ }_{74} \mathrm{~W}^{180}$ & 0.1556 & 102 & 1.65 & 41.4 & & & \\
\hline $\mathrm{W}^{182}$ & 0.1428 & 100 & 1.75 & 49.1 & 7.0 & 49 & 1.00 \\
\hline$W^{184}$ & 0.1304 & 112 & 1.70 & 45.7 & 6.7 & 51 & 1.12 \\
\hline$W^{186}$ & 0.1183 & 124 & 1.67 & 43.8 & 5.9 & 50 & 1.14 \\
\hline
\end{tabular}


moments for a number of even-even nuclei. The values so obtained are shown in Table I. Nuclei from Gd ${ }^{154}$ to $\mathrm{W}^{186}$ have been included in the table. It is probably true that pure rotational spectra exist for some nuclei at either end of the indicated region of $A$. However, there seems to be conflicting evidence in certain cases, and it is felt that all the available evidence points to pure rotational spectra in the region indicated above. The values of $k$ are calculated from the number of nucleons in each case.

The present concept of a fractional mass (or charge) participating in nuclear rotations has a natural extension to the interpretation of the data on electrodynamic transitions between nuclear ground states and rotational levels. This extension follows as a consequence of the assumption that the mass and charge densities of nuclear matter are uniform. Hence, if it is assumed that only a certain fraction of the mass participates in collective motion, then for the sake of consistency it should be assumed that only the same fraction of the charge participates in the motion. This emphasizes the fact that the prescription proposed for the determination of the mass fraction in terms of the excess of particles or holes outside filled major shells must be interpreted as providing an estimate of only the magnitude of the fraction and not its character in the sense of a dependence upon the particular kind of particles outside the major shells in a given case.

Since both rates for radiative transitions ${ }^{1}$ from and cross sections for Coulomb excitation ${ }^{2}$ of nuclear rotational levels are proportional to the nuclear current, it is seen that the interpretation of the data relative to such processes will be explicitly dependent on the charge fraction participating in collective motion. It has been shown ${ }^{3}$ that for the case of the uniformly-charged axially-symmetric rigid rotator considered in the present work, the values of $Q_{0}$ determined from the measured lifetimes of first-excited $2^{+}$states of even-even nuclei in the rare-earth region as given by Bohr and Mottelson ${ }^{4}$ must be divided by $k$. It is expected that the same modification would hold in the determination of $Q_{0}$ 's from Coulomb-excitation crosssection data.

The intrinsic quadrupole moments obtained from transition data are shown in Table $\mathrm{I}$. The values given in the second column as $Q_{0}(\mathrm{~B}-\mathrm{M})$, except those for the tungsten isotopes, are those listed on page 18 of Bohr and Mottelson's paper. ${ }^{4}$ The values given for tungsten have been obtained with separated isotopes by McGowan and Stelson. ${ }^{5}$ The numbers listed in the table have been obtained from both Coulomb-excitation cross-section data and from lifetime data.

It is of interest to point out that the precision of the measurement of the energy levels is of the order of a few percent and that of the Coulomb-excitation cross sections, considering the uncertainties in making absolute intensity measurements and the uncertainties in the values of total conversion coefficients, is of the order of $30 \%$.

The authors wish to express their gratitude to Professor L. W. Nordheim for his advice and encouragement. It is a pleasure to acknowledge the assistance of Virginia C. Klema, who checked the calculations given above.

${ }^{1}$ J. M. Blatt and V. F. Weisskopf, Theoretical Nuclear Physics (John Wiley and Sons, Inc., New York, 1952).

2 Biedenharn, McHale, and Thaler, Phys. Rev. 100, 376 (1955).

${ }^{3}$ R. K. Osborn and L. C. Biedenharn (unpublished).

${ }^{4}$ Aage Bohr and Ben Mottelson, Kgl. Danske Videnskab. Selskab, Mat.-fys. Medd. 30, No. 1 (1955).

${ }^{5}$ F. K. McGowan and P. H. Stelson (private communication).

\section{Observation of Nuclear Magnetic Resonances via the Electron Spin Resonance Line}

\author{
G. FEHER \\ Bell Telephone Laboratories, Incorporated, \\ Murray Hill, New Jersey \\ (Received June 15, 1956)
}

$T$

HE double-frequency resonance method reported recently in connection with a nuclear polarization scheme $^{1}$ has been extended to observe nuclear transitions and thereby determine hyperfine interactions and nuclear $g$ values.

The method is illustrated for the simple case $I=\frac{1}{2}$, $J=\frac{1}{2}$, in which the hyperfine structure is resolved. The transitions induced by the microwave field of frequency $\nu_{e}$ are indicated by arrows in Fig. 1. The amplitude of the signal due to these transitions is proportional to the difference in population in levels $A$ and $A^{\prime}$. If we partially saturate this resonance the population difference will be diminished and the signal reduced. By inducing the nuclear transitions $h \nu_{N}$ (see Fig. 1), we may either equalize the populations in levels $A$ and $B$ (by saturating these transitions) or reverse the populations (by an adiabatic fast passage).

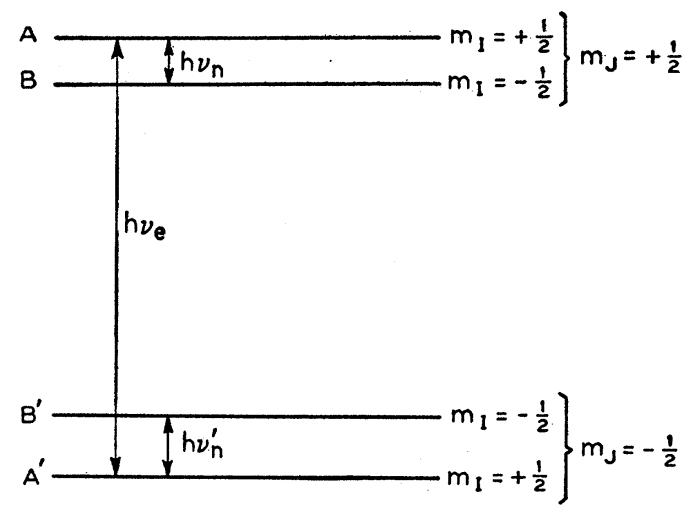

FIG. 1. Energy levels of a system with $I=\frac{1}{2}, J=\frac{1}{2}$. Arrows indicate transitions which were induced in the phosphorus-doped silicon sample. 